\title{
Neue Indolinbasen
}

von

\section{Alois Plangger.}

(Vorgelegt in der Sitzung am 6. April 1905.)

Zur Fortsetzung der von Prof. Dr. K. Brunner unter dem Titel »Synthese von Indolinbasen vor einigen Jahren veröffentlichten Abhandlung ${ }^{1}$ suchte ich das von ihm aufgefundene Verfahren zur Darstellung solcher Basen an dem Orthotolylhydrazon des Methylisopropylketons zu erproben. Das aus dem käuflichen Hydrochlorid dargestellte Orthotolylhydrazin habe ich mit wenig mehr als der molekularen Menge an Methylisopropylketon versetzt und nach eintägigem Stehen bei Zimmertemperatur zur Vollendung der Hydrazonbildung durch $11 / 2$ Stunden am Rückflußkühler auf dem Wasserbade erwärmt. Das mit entwässertem Natriumsulfat getrocknete Hydrazon wurde zur Reinigung im Vakuum destilliert, wobei es unter zirka $20 \mathrm{~mm}$ Druck bei $154-158^{\circ}$ überging. Nach Brunner's Verfahren konnte nun die Bildung einer Indolinbase unter Abspaltung von Ammoniak entweder durch die Einwirkung von alkoholischer Jodwasserstoffsäure oder durch Erwärmen mit alkoholischer Chlorzinklösung oder mit alkoholischer Lösung von Zinnchlorür und Salzsäure eintreten. Nach diesbezüglichen Vorversuchen trat bei Proben mit diesen Reagentien Ammoniak aus, jedoch konnte die Isolierung der neugebildeten Indolinbase nur mit Jodwasserstoffsäure oder mit Zinnchlorür erreicht werden.

Zur Behandlung mit alkoholischer Jodwasserstoffsäure wurden je $3 g$ Orthotolylhydrazon mit einem abgekühlten Gemisch von $3 g$ farbloser Jodwasserstoffsäure (spez. Gew. 2)

1 Monatshefte für Chemie, 21, p. 156 (1900). 
und $6 \mathrm{~cm}^{3}$ Alkohol $(96 \%)$ gemengt. Nach dreitägiger Einwirkung bei Zimmertemperatur wurde mit Eiswasser gekühlt und die zehnfache Volummenge reinen Äthers zugesetzt. Hiedurch schied sich eine ölige Flüssigkeit ab, die bei Winterkälte nach einiger Zeit seidenglänzende Kristalle abschied, die mit Ather gewaschen und aus Chloroform umkristallisiert wurden. Die Kristalle sind in Wasser oder Weingeist sehr leicht löslich. Aus kochender Chloroformlösung scheiden sie sich beim Erkalten in Form von Nadeln aus, die bei $185.5^{\circ} \mathrm{C}$. zu einer braunen Flüssigkeit schmelzen. Das lufttrockene Salz erleidet über Schwefelsäure im Vakuum keinen Gewichtsverlust.

$0 \cdot 2054 \mathrm{~g}$ Substanz gabon $0.3601 \mathrm{~g}$ Kohlenoxyd und $0 \cdot 1047 \mathrm{~g}$ Wasser. $0.2625 g$ Substanz gaben $0.2025 g$ Jodsilber.

In 100 Teilen:

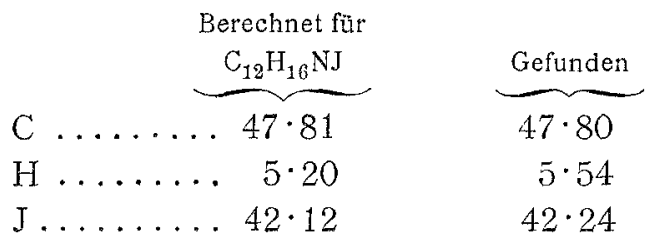

Weil die Ausbeute an diesem Jodhydrat eine ungleichmäßige und geringe war, so wurde für weitere Versuche das Verfahren mit Zinnchlorür, welches sogleich ein an der Luft unverändert haltbares Zinnchlorürdoppelsalz lieferte, vorgezogen. Je $3 g$ Hydrazon habe ich mit einer abgekühlten Lösung von $3 \mathrm{~g}$ Zinnchlorür (Stannum chloratum fusum) in $6 \mathrm{~g}$ Alkohol $(96 \%)$ und $2 g$ Salzsäure 1.19 vermischt und am Rückflußkühler auf dem Wasserbade eine Stunde hindurch erwärmt. Nach längerem Stehen in der Kälte, schneller beim Schütteln, schieden sich lange Kristallnadeln $a b$, die von der darüberstehenden Lösung getrennt und mit Äther gewaschen wurden. Aus der abgegossenen Lösung konnten durch Zusatz von Salzsäure und Äther beim Stehenlassen in der Kälte noch weitere Mengen von diesen Kristallen erhalten werden. Die Ausbeute an diesem allerdings noch mit Chlorammonium verunreinigten Doppelsalz erreichte die nach der Berechnung für 
eine vollständige Überführung in Indolinbase mögliche Menge. Das Salz konnte jedoch nicht durch Umkristallisieren gereinigt werden.

\section{Base $\mathrm{C}_{12} \mathrm{H}_{15} \mathbf{N}$.}

Zur Herstellung der Base wurde deshalb das unmittelbar gewonnene Zinndoppelsalz mit Kalilauge im Überschuß zersetzt, die Base im Wasserdampfstrome überdestilliert und aus dem Destillat durch Ausschütteln mit Äther aufgenommen. Die ätherische, mit entwässerter Pottasche getrocknete Lösung ließ beim Abdestillieren die Base als gelbes Öl zurück, das sofort durch Destillation im Vakuum gereinigt wurde. Die Base destillierte unter zirka $20 \mathrm{~mm}$ Druck bei $158^{\circ}$ über und stellte ein nur schwach gelb gefärbtes Öl dar, das sofort in Glasröhren eingeschmolzen wurde, weil es sonst an der Luft alsbald eine braune Färbung annahm. Die Base blieb auch bei niedriger Temperatur flüssig.

$0 \cdot 3711 \mathrm{~g}$ Substanz gaben $1 \cdot 1325 \mathrm{~g}$ Kohlendioxyd und $02839 \mathrm{~g}$ Wasser.

$0.3607 \mathrm{~g}$ Substanz gaben $25 \cdot 9 \mathrm{~cm}^{3}$ feuchten Stickstoff bei $722 \mathrm{~mm}$ Barometerstand und $13^{\circ}$.

In 100 Teilen:

\begin{tabular}{ccc}
\multicolumn{4}{c}{$\begin{array}{c}\text { Berechnet für } \\
\mathrm{C}_{12} \mathrm{H}_{15} \mathrm{~N}\end{array}$} & $\underbrace{\text { Gefunden }}_{83 \cdot 21}$ \\
$\mathrm{H} \ldots \ldots \ldots$ & $83 \cdot 23$ \\
$\mathrm{~N} \ldots \ldots \ldots$ & 8.66 & 8.50 \\
& 8.12 & 8.02
\end{tabular}

\section{Salze der Base.}

Bei dem Versuche, kristallisierte Salze der Base darzustellen, zeigte sich, daß dieselben sehr leicht löslich sind und an der Luft bald eine dunkle Färbung annehmen. Es konnten daher aus wässerigen Lösungen in keinerlei Weise kristallisierte Salze in reiner Form erhalten werden. Auch aus alkoholischer Lösung konnte ich nur das vorher erwähnte Jodhydrat kristallisiert erhalten. In ätherischer Lösung fällt auf Zusatz von ätherischer Pikrinsäurelösung, die mit wenig Petroläther versetzt war, ein hellgelbes, krisțallisiertẹs Pikrat, das nach 
dem Lösen in heißem Alkohol beim Erkalten desselben auskristallisierte. Es schmilzt bei $174^{\circ}$ unter Zersetzung.

$0.2575 \mathrm{~g}$ Substanz gaben $0.5450 \mathrm{~g}$ Kohlensäure und $0.1112 \mathrm{~g}$ Wasser. $0.2889 \mathrm{~g}$ Substan $z$ gaben $37.25 \mathrm{~cm}^{3}$ feuchten Stickstoff bei $727 \mathrm{~mm}$ Barometerstand und $16^{\circ}$.

In 100 Teilen: Berechnet für

\begin{tabular}{|c|c|c|}
\hline & $\mathrm{C}_{12} \mathrm{H}_{15} \mathrm{~N} \cdot \mathrm{C}_{6} \mathrm{H}_{3} \mathrm{O}\left(\mathrm{NO}_{2}\right)_{3}$ & Gefunden \\
\hline & $\ldots 57 \cdot 75$ & $57 \cdot 7$ \\
\hline $\mathrm{H}$ & $4 \cdot 81$ & $4 \cdot 8$ \\
\hline & $\ldots 15 \cdot 00$ & $14 \cdot 68$ \\
\hline
\end{tabular}

\section{Base $\mathrm{C}_{13} \mathrm{H}_{17} \mathrm{~N}$.}

Bei der Behandlung mit Jodmethyl addiert die früher erwähnte Base dieses schon beim Erwärmen auf dem Wasserbade am Rückflußkühler. Nach vierstündigem Erwärmen scheidet sich ein rötlich gefärbtes Jodid $a b$, das sich von dem früher erwähnten Jodhydrat durch die Schwerlöslichkeit in Alkohol unterscheidet. In der Lösung ist nur wenig von diesem schwerlöslichen Jodid enthalten, hingegen findet sich das leicht lösliche Jodhydrat der früheren Base, nach deren Isolierung bei abermaliger Behandlung mit Jodmethyl fast nur mehr das schwerlösliche Jodid erhalten wurde. Durch Umkristallisieren aus kochendem Alkohol unter Zusatz von Tierkohle wurde dieses Jodid in Form von weißen Kristallblättchen erhalten, die bei $212^{\circ}$ unter Zersetzung schmelzen.

$0.2200 \mathrm{~g}$ Substanz gaben $0.3997 \mathrm{~g}$ Kohlendioxyd und $0.1129 \mathrm{~g}$ Wasser.

$0 \cdot 2533 \mathrm{~g}$ Substanz gaben $0 \cdot 1902 \mathrm{~g}$ Jodsilber.

In 100 Teilen:

\begin{tabular}{|c|c|c|}
\hline & $\begin{array}{l}\text { Berechnet für } \\
\mathrm{C}_{1 \mathbf{s}} \mathrm{H}_{18} \mathrm{NJ}\end{array}$ & Gefunden \\
\hline $\mathrm{C}$ & $49 \cdot 54$ & $49 \cdot 52$ \\
\hline $\mathrm{H} \ldots \ldots$ & $5 \cdot 72$ & $5 \cdot 7$ \\
\hline J....... & . $40 \cdot 26$ & $40 \cdot 32$ \\
\hline
\end{tabular}

Um die Base zu gewinnen, zersetzte ich ihr Jodid mit einem Überschuß von Kalilauge und nahm sie in Äther auf. 
Die mit entwässerter Pottasche getrocknete Lösung hinterließ die Base als gelblichrotes Ö1, welches sofort durch Destillation im Vakuum gereinigt wurde. Unter zirka $20 \mathrm{~mm}$ Druck destillierte sie bei $138^{\circ}$ als vollkommen farblose Flüssigkeit über, welche für die Analyse in entsprechende Glasröhrchen eingeschlossen wurde.

$0.2305 \mathrm{~g}$ Substanz gaben $0.7047 \mathrm{~g}$ Kohlendioxyd und $0.1884 \mathrm{~g}$ Wasser.

$0.1761 \mathrm{~g}$ Substanz gaben $12.6 \mathrm{~cm}^{2}$ feuchten Stickstoff bei $713 \mathrm{~mm}$ Barometerstand und $15^{\circ}$.

In 100 Teilen:

\begin{tabular}{|c|c|c|}
\hline & $\begin{array}{l}\text { Berechnet für } \\
\mathrm{C}_{13} \mathrm{H}_{17} \mathrm{~N}\end{array}$ & Gefunden \\
\hline C & $83 \cdot 37$ & $83 \cdot 38$ \\
\hline H. . & $9 \cdot 14$ & $9 \cdot 08$ \\
\hline$N \ldots \ldots$ & $7 \cdot 49$ & $7 \cdot 84$ \\
\hline
\end{tabular}

Auch diese Base zeigte bei niedriger Temperatur keine Neigung zur Kristallisation. In offenen Gefäßen nimmt sie an der Luft alsbald eine intensiv karminrote Färbung an. Ihre Salze kristallisieren gut und sind daher leichter erhältlich als die der vorher erwähnten Base. So fällt das Pikrat nach der Vereinigung der ätherischen Lösungen der Pikrinsäure mit der Base sofort kristallisiert heraus. Es stellt nach dem Umkristallisieren aus heißem Alkohol zitronengelbe Nadeln dar, die bei $165^{\circ}$ schmelzen.

Die salzsaure Lösung der Base gibt mit konzentrierter Eisenchloridlösung eine Trübung, nach Zugabe von konzentrierter Salzsäure einen kristallinischen Niederschlag, der aus einem Eisendoppelsalz besteht; dasselbe ist in heißem Alkohol löslich und scheidet sich aus diesem beim Erkalten kristallisiert ab. Es stellt so weißlich-braune, zarte Nadeln vom Schmelzpunkte $113^{\circ}$ dar.

Das Orthotolylhydrazon des Isopropylmethylketons geht also unter denselben Bedingungen, bei denen Brunner die E. Fischer'sche Base und Pr-1-Phenyl-3 - 3-Dimethyl-2-Methylenindolin aus den entsprechenden Hydrazonen erhalten hat, in ein $B$-1, $P r-3 \cdot 3$-Dimethyl-2-Methylindolenin über. Sie ent- 
spricht in ihrem Verhalten der von G. Plancher ${ }^{1}$ nach Brunner's ${ }^{2}$ Verfahren aus dem Phenylhydrazon des Isopropyl-, methylketons dargestellten Indoleninbase. Wie diese mit Jodmethyl die Fischer'sche Base liefert, so nimmt auch das $B$-1, Pr-33-Dimethyl-2-Methylindolenin Jodmethyl schon beim Kochen am Rückflußkühler auf, geht damit in das Jodid des $B$-1, Pr-1-n-Methyl-3-3-Dimethyl-2-Methylenindolin über.

Die aus diesem Jodid mit Laugen abgeschiedene Base zeigt wie die Fischer'sche Base das charakteristische Verhalten, sich an der Luft rot zu färben, ein in Alkohol schwer lösliches Jodid und ein mit konzentrierter Salzsäure fällbares Eisendoppelsalz zu geben. Die Stellung der Methylgruppe im Benzolkern und der übrigen Gruppen im Pyrrolkern sind durch die Synthese und die vollkommene Analogie der Fischer'schen Base eindeutig bestimmt, so daß für diese Base die folgende Strukturformel in Übereinstimmung mit obiger Nomenklatur aufgestellt werden kann.<smiles>C=CC1C2CCCCC2C(C)(C)C1C</smiles>

Der Versuch, eine Base aus einem Orthotolylhydrazon des Isopropylmethylketons nach obigem Verfahren darzustellen, schien auch mit Rücksicht darauf von Interesse, weil das Vorhandensein einer Methylgruppe in der Orthostellung möglicherweise auch zu einem Tetrahydrochinolinderivat hätte führen können. Die Bildung einer solchen Base wurde jedoch nicht beobachtet, sondern eben nur eine Indolinbase erhalten.

1 Chem. Ztg., 22, p. 37-38 (1898).

3 Monatshefte f. Chemie, 16, p. 849 (1895). 